\title{
Spin fluids in van Stockum cylinders
}

\author{
J P Krisch $\dagger$ and L L Smalley $\ddagger \S$ \\ $\dagger$ Department of Physics, University of Michigan, Ann Arbor, MI 48109-1120, USA \\ \$ES65/Space Science Laboratory, Marshall Space Flight Center, AL 35812, USA \\ $\S$ Department of Physics, University of Alabama in Huntsville, Huntsville, AL 35899, USA
}

Received 2 August 1989

\begin{abstract}
Within general relativity, the stress-energy content of van Stockum's twodimensional dust interior is extended to include a two-dimensional spin fluid. The metric and spin-fluid parameters are expressed in terms of one generating function. As an application, a rotating cloud of spin fluid is discussed.
\end{abstract}

\section{Introduction}

Rotating fluids are of great interest in relativistic physics. They are used in stellar and cosmological models, in experimental gravitational detection systems and in developing idealised models to study specific aspects of the matter-curvature interaction.

In 1937, van Stockum [1] solved a problem of the latter type. He gave the metric and matter content of an infinite rotating cylindrical distribution of matter. The matter content and metric potentials of his solution were two dimensional, depending on the radial coordinate $r$ and $z$ in a cylindrical $(0, r, \phi, z)$ system. The solution was stated in terms of a function $\Psi(r, z)$ obeying the equation

$$
\Psi_{r r}-\Psi_{r} / r+\Psi_{z z}=0
$$

where $\Psi_{i}$ represents the ordinary derivative with respect to the $i$ th coordinate. The van Stockum metric is

$$
\mathrm{d} s^{2}=-\mathrm{d} t^{2}-2 k \mathrm{~d} \phi \mathrm{d} t+\left(D^{2}-k^{2}\right) \mathrm{d} \phi^{2}+\mathrm{e}^{\mu}\left(\mathrm{d} r^{2}+\mathrm{d} z^{2}\right)
$$

with

$$
\mu_{r}=(1 / 2 D)\left(\Psi_{z}^{2}-\Psi_{r}^{2}\right) \quad k=\Psi \quad \mu_{z}=(-1 / D) \Psi_{r} \Psi_{z} .
$$

From the field equations, $D$ is found to be a solution of the Cartesian Laplace equation

$$
D_{r r}+D_{z z}=0
$$

The solution commonly chosen is $D=r$.

The fluid in the rotating cylinder is pressure-free dust and has energy density

$$
\varepsilon=r^{-2} \mathrm{e}^{-\mu}\left(\Psi_{r}^{2}+\Psi_{z}^{2}\right) \text {. }
$$

This solution is interesting when compared with the Newtonian rotating cylinder. In the relativistic cylinder there can be a density gradient along the $z$ axis [2] which is absent in the Newtonian cylinder. The interior and some matching exteriors can develop closed timelike lines [3] in common with the Kerr and Godel exteriors [4]. The matter content of some of the interior solutions exhibit a directional singularity $[5,6]$ which may represent an extended axial source. 
In this paper, we extend the energy content of van Stockum's two-dimensional dust interior to include a two-dimensional stress-energy content with an intrinsic spin density. The corresponding metric is obtained. As in the original development, all metric potentials and fluid parameters are expressed in terms of one generating function. The formalism is developed in the next section. This extension, which widens the applicability of the van Stockum spacetimes, is also useful in interpreting the source structure of apparent axial matter singularities. As an application we apply the formalism to a rigidly rotating cloud of spin fluid. This application is in $\S 2.3$.

\section{Extension of the van Stockum solution}

\subsection{Field equations}

Rotation can be included in relativistic calculations by allowing a metric component $g_{0 \mu}$. The fluid rotation introduced in this way is described by the vorticity tensor [7]

$$
w_{\mu \nu}=U_{[\mu ; \nu]}+\dot{U}_{[\mu} U_{\nu]}
$$

where $U_{\mu}$ is the fluid velocity and $\dot{U}_{\mu}=U_{\mu ; \nu} U^{\nu}$ the acceleration. For the metric described by equation (2) the velocity of the fluid can be described by

$$
U^{\mu}=(1,0,0,0) \quad U_{\mu}=(-1,0,-k, 0) .
$$

The fluid acceleration is zero.

In addition to the angular velocity described by the vorticity, there is an angular velocity related to an intrinsic spin density $S_{\mu \nu}$. The rotation associated with the spin density can be described by the tensor [8]

$$
\tilde{w}_{\mu \nu}=\dot{A}_{\mu}^{(i)} A_{\nu(i)}
$$

where $A_{\mu}^{(i)}$ is an orthonormal tetrad chosen so that $A_{(0)}^{\mu}$ lies along the velocity $U^{\mu}$. Tetrad indices are in parentheses $(i)=(0,1,2,3)$ and are raised and lowered with the Minkowski metric $(-+++)$. Coordinate indices are not in parentheses, $\mu=(0, r, \phi, z)$.

For the metric described by equation (2), a possible tetrad is [9]

$$
\begin{aligned}
& A_{(0)}^{\mu}=(1,0,0,0) \quad A_{(1)}^{\mu}=\left(0, \mathrm{e}^{-\mu / 2}, 0,0\right) \\
& A_{(2)}^{\mu}=(-k / D, 0,1 / D, 0) \quad A_{(3)}^{\mu}=\left(0,0,0, \mathrm{e}^{-\mu / 2}\right) .
\end{aligned}
$$

This particular tetrad diagonalises the metric. For time-independent problems, the vorticity described by equation (8) is independent of the tetrad choice. For the unaccelerated fluid of metric (2) the spin and fluid vorticity have the same form

$$
\begin{aligned}
& w_{r \phi}=\tilde{w}_{r \phi}=\frac{1}{2} k_{r} \\
& w_{z \phi}=\tilde{w}_{z \phi}=\frac{1}{2} k_{z} .
\end{aligned}
$$

The spin density $S_{\mu \nu}$ defines a density of angular momentum throughout the fluid. It is constrained by the Frenkel condition [10]

$$
U^{\mu} S_{\mu \nu}=0 \text {. }
$$

A spin vector $S^{\mu}$ can be associated with the spin density

$$
S^{\mu}=\frac{1}{2} \varepsilon^{\mu \nu p \sigma} U_{\nu} S_{p \sigma}(-g)^{-1 / 2} .
$$


Ray and Smalley [11] have developed a stress-energy tensor that includes an intrinsic spin density. The stress-energy tensor can be written as the sum of two parts

$$
T^{\mu \nu}=T_{(\text {fluid })}^{\mu \nu}+T_{(\text {spin })}^{\mu \nu}
$$

where $T_{\text {(fluid) }}^{\mu \nu}$ is the perfect fluid stress-energy tensor

$$
T_{\text {(fluid) }}^{\mu \nu}=(\varepsilon+p) U^{\mu} U^{\nu}+p g^{\mu \nu}
$$

$\varepsilon$ is the energy density and $p$ the pressure.

The spin-fluid portion of the stress-energy tensor is

$$
T_{(\mathrm{spin})}^{\mu \nu}=2 U^{(\mu} S^{\nu) \sigma} \dot{U}_{\sigma}+\left(U^{(\mu} S^{\nu) \sigma}\right)_{; \sigma}-\tilde{w}^{\sigma(\mu} S^{\nu)} \sigma .
$$

This stress-energy tensor was developed within the context of the extended EinsteinCartan relativity but can be used in the torsion-free spacetimes of general relativity [12]. We will use this stress-energy tensor to write the tetrad indexed field equations

$$
G_{(i j)}=A_{(i)}^{\mu} A_{(j)}^{\nu} G_{\mu \nu}=A_{(i)}^{\mu} A_{(j)}^{\nu} T_{\mu \nu}=T_{(i j)} .
$$

We take $8 \pi G=1$.

We will include only spin components along the $r$ and $z$ axes since consistency conditions on the $\phi$ spin component require an irrotational fluid. The field equations are

$$
\begin{aligned}
& \text { (00): } \frac{3 k_{r}^{2}}{4 D^{2}}+\frac{3 k_{z}^{2}}{4 D^{2}}-\frac{\mu_{z z}}{2}-\frac{\mu_{r r}}{2}-\frac{D_{r r}}{D}-\frac{D_{z z}}{D}=\varepsilon \mathrm{e}^{\mu}+S_{r \phi} \frac{k_{r}}{D^{2}}+S_{z \phi} \frac{k_{z}}{D^{2}} \\
& \text { (11): } \frac{D_{r} \mu_{r}}{2 D}+\frac{k_{r}^{2}}{4 D^{2}}+\frac{D_{z z}}{D}-\frac{D_{z} \mu_{z}}{2 D}-\frac{k_{z}^{2}}{4 D^{2}}=p \mathrm{e}^{\mu}+S_{r \phi} \frac{k_{r}}{D^{2}} \\
& \text { (22): } \frac{k_{r}^{2}}{4 D^{2}}+\frac{\mu_{r r}}{2}+\frac{k_{z}^{2}}{4 D^{2}}+\frac{\mu_{z z}}{2}=p \mathrm{e}^{\mu}+S_{r \phi} \frac{k_{r}}{D^{2}}+S_{z \phi} \frac{k_{z}}{D^{2}} \\
& \text { (33): } \frac{D_{r r}}{D}-\frac{D_{r} \mu_{r}}{2 D}-\frac{k_{r}^{2}}{4 D^{2}}+\frac{D_{z} \mu_{z}}{2 D}+\frac{k_{z}^{2}}{4 D^{2}}=p \mathrm{e}^{\mu}+S_{z \phi} \frac{k_{z}}{D^{2}} \\
& \text { (02): }\left(\frac{k_{r}}{2 D}\right) r+\left(\frac{k_{z}}{2 D}\right) z=\left(\frac{S_{r \phi}}{2 D}\right) r+\left(\frac{S_{z \phi}}{2 D}\right) z \\
& r \theta: \frac{-D_{r z}}{D}+\frac{\mu_{r} D_{z}}{2 D}+\frac{\mu_{z} D_{r}}{2 D}+\frac{k_{r} k_{z}}{2 D^{2}}=\frac{S_{r \phi} k_{z}}{2 D^{2}}+\frac{S_{z \phi} k_{r}}{2 D^{2}} .
\end{aligned}
$$

\subsection{The extended solution}

Adding the (11) and (33) components of the field equations we obtain

$$
\frac{D_{r r}}{D}+\frac{D_{z z}}{D}=2 p \mathrm{e}^{\mu}+\frac{S_{r \phi} k_{r}}{D^{2}}+\frac{S_{z \phi} k_{z}}{D^{2}} .
$$

This equation is one of the major departures from the van Stockum solutions for spinless dust. When spin is included, this is no longer a Laplace but a Poisson equation. Clearly a wealth of different fluids can be generated for study by using pressure and spin as a source for a new $D$ function. For example, one could specify spinning dust and generate the $D$ function corresponding to a spin-vorticity source. Since the purpose of this calculation is to extend the original van Stockum fluid content to include spin 
in a way that allows comparison with existing metric solutions, we shall retain the harmonic van Stockum choice of

$$
D=r \text {. }
$$

This choice will produce metrics closely related to existing cylindrical spacetimes and permit a comparison of the fluid contents. It also creates a spin-related pressure

$$
p \mathrm{e}^{\mu}=S_{\phi r} k_{r} / 2 r^{2}+S_{\phi z} k_{z} / 2 r^{2} .
$$

The generating function for the solution comes from the $(02)$ equation. Define a function $\Psi(r, z)$ such that

$$
\begin{aligned}
& \bar{\Psi} r=k_{r}-S_{r \phi} \\
& \bar{\Psi}_{z}=k_{z}-S_{z \phi} .
\end{aligned}
$$

Equation (21) becomes

$$
\bar{\Psi}_{r r}-\bar{\Psi} / r+\bar{\Psi}_{z z}=0
$$

which is identical to the equation for the van Stockum generating function $\Psi(r, z)$, equation (1). We will drop the bar and take the generating functions to be the same. To achieve consistency with the field equations, the spin density and the function $k(r, z)$ can be separately proportional to $\Psi(r, z)$

$$
k=\alpha \Psi \quad S_{r \phi}=-\beta \Psi, \quad S_{z \phi}=-\beta \Psi_{z} \quad \alpha=-\beta+1 .
$$

Substituting into the field equations, one easily finds the extended van Stockum relations

$$
\mu_{z}=-\left(1-\beta^{2}\right) \frac{\Psi_{r} \Psi_{z}}{r} \quad k=\alpha \Psi \quad \mu_{r}=\frac{\left(\Psi_{z}^{2}-\Psi_{r}^{2}\right)}{2 r}\left(1-\beta^{2}\right)
$$

with fluid content

$\varepsilon \mathrm{e}^{\mu}=(2+\beta)\left[(1-\beta) / 2 r^{2}\right]\left(\Psi_{r}^{2}+\Psi_{z}^{2}\right) \quad p \mathrm{e}^{\mu}=\left(+\alpha \beta / 2 r^{2}\right)\left(\Psi_{r}^{2}+\Psi_{z}^{2}\right)$

$\mathrm{e}^{\mu} S^{2}=S^{r} S_{r}+S^{z} S_{z}=\left(\beta^{2} / r^{2}\right)\left(\Psi_{r}^{2}+\Psi_{z}^{2}\right)$

Equations (29)-(31) are the extended van Stockum solution. They will describe a cylindrical distribution of fluid with metric potentials (30). In order to describe a physical fluid, $\beta$ must obey the constraint

$$
0 \leqslant \beta<1 \text {. }
$$

The extended solution differs from the dust solution in the presence of a non-zero spin density and associated pressure. The energy and metric potentials are scaled away from their dust values. The spinless but rotating dust solution is obtained in the limit $\beta \rightarrow 0$.

\subsection{A rigidly rotating spin-fluid cloud}

A choice that has often been made for $\Psi$ is

$$
\Psi(r, z)=-2 h r^{2}\left(r^{2}+z^{2}\right)^{-3 / 2} .
$$

This choice is motivated by the fact that $\Psi$ can be related to a function $\xi$ satisfying a cylindrical Laplace equation [13]

$$
\xi_{r r}+\xi_{r} / r+\xi_{z z}=0 \quad \Psi=r \xi_{r} .
$$


The particular choice (33) corresponds to

$$
\xi=2 h\left(r^{2}+z^{2}\right)^{-1 / 2} \text {. }
$$

Using this generating function, Bonnor [2] has developed a model for a rigidly rotating cylindrical dust cloud. The dust in this model has a density gradient along the $z$ axis which is absent in the Newtonian problem. It also has a directional singularity [5] that might signal an extended source. Islam [6] has discussed this problem with relation to a charged dust cylinder.

Using the generating function (33), the spin-fluid extended metric potentials are

$$
\mu=\frac{\left(1-\beta^{2}\right) h^{2} r^{2}\left(r^{2}-8 z^{2}\right)}{2\left(r^{2}+z^{2}\right)^{4}} \quad k=\frac{-2 \alpha h r^{2}}{\left(r^{2}+z^{2}\right)^{3 / 2}} .
$$

The parameters of the fluid that is the source for this metric are

$$
\begin{aligned}
& \varepsilon=(2+\beta)(1-\beta) x(r, z) \quad p=+\alpha \beta x(r, z) \\
& S^{2}=\frac{1}{2} \beta^{2} x(r, z) \quad x(r, z)=2 h^{2}\left(r^{2}+4 z^{2}\right) \mathrm{e}^{-\mu}\left(r^{2}+z^{2}\right)^{-4} .
\end{aligned}
$$

Direct substitution of (36) into equations (17)-(22) easily verifies this matter content. The directional singularity found by Bonnor for dust is also present in the spin fluid. The fluid parameters, pressure, energy and total spin all depend on the same function $x(r, z)$, which has different limits depending on the line of approach to the origin. A similar extended source behaviour has also been discussed by Stewart et al [14] within the context of a toroidal topology.

In Bonnor's calculation, the metric source was identified as a rotating cloud of dust with a spinning singularity at the origin. The explicit inclusion of spin, while eliminating the dust, makes plausible the interpretation of the axial singularity as an object with spin since there is now a definite spin form in the fluid that has the same directional singularity structure as the energy density. The presence of a $z$-dependent pressure induced by spin helps explain the support of the fluid above the origin plane [15]. In the $r-\phi$ plane, the fluid and metric are the simple one-dimensional van Stockum interior with only a $z$ component of spin. This case has been discussed by Krisch and Smalley [16].

Spin fluids in the general extension described by equations (29)-(31) have an equation of state

$$
\varepsilon=\frac{(2+\beta)}{\beta} p
$$

For the range of allowed $\beta$ this gives

$$
\varepsilon>3 p \text {. }
$$

Bayin [17] has shown that equations of state of this form can be obtained for a lattice of baryons at densities $\rho \geqslant 10^{15} \mathrm{~g} \mathrm{~cm}^{-3}$ with no violations of causality. In light of this, one might possibly interpret the complete metric source as a structured cloud of baryons with an extended axial spinning source.

In conclusion, we have developed a formalism that allows a two-dimensional spin fluid to be used as a source for the metric of a cylindrical matter distribution. This extension can be used to provide additional information on the source structure of existing solutions. By relaxing the harmonic constraint on the $D$ function, new interiors, unobtainable for the dust cylinder, can be studied. 
One possible physically interesting application of the spin-fluid formalism is to the development and behaviour of vortex distributions in superfluids. Superfluids appear in many contexts in relativistic problems. For example, they are used in the design of gravitational detectors [18], an experiment with cosmological significance involving the appearance of vortex lines in superfluid ${ }^{4} \mathrm{He}$ has been discussed by Zurek [19] and superfluids have been suggested as a component of neutron-star interiors [20]. In problems with vortices, the vortex distribution could be easily modeled by a spin fluid density. The symmetry of the infinite cylinder is appropriate to the experimental set-up of rotating superfluids in the laboratory and might possibly be applied to other symmetries in regions far from boundaries.

Spin, in all of its aspects, is becoming increasingly important in physics. Applications of a general relativistic spin-fluid formalism to problems in condensed-matter physics will inevitably lead to new insights about the interaction of spin and geometry.

\section{References}

[1] van Stockum W 1937 Proc. R. Soc. Edinburgh 57135

[2] Bonnor W B 1977 J. Phys. A: Math. Gen. 101673

[3] Bonnor W B 1980 J. Phys. A: Math. Gen. 132121

[4] Raychaudhuri A K 1979 Theoretical Cosmology (Oxford: Clarendon) p 94

[5] Gautreau R and Anderson J L 1967 Phys. Lett. 25291

[6] Islam J N 1977 Proc. R. Soc. A 353523

[7] Stephani H 1982 General Relativity (Cambridge: Cambridge University Press) p 166

[8] Halbwachs F 1960 Prog. Theor. Phys. 24291

[9] This tetrad corrects a typographical error in reference [16]. The labellings of [16] are unchanged.

[10] Frenkel J 1926 Z. Phys. 37243

[11] Ray J R and Smalley L L 1983 Phys. Rev. D 27 1383; 1982 Phys. Rev. Lett. 49 1059; 1983 50 626(E)

[12] Ray J R and Smalley L L 1982 Phys. Rev. D 262619

[13] Islam J N 1985 Rotating Fields in General Relativity (Cambridge: Cambridge University Press) p 30

[14] Stewart B W, Witten L and Papadopoulos D 1987 Gen. Rel. Grav. 19827

[15] Islam J N 1985 Rotating Fields in General Relativity (Cambridge: Cambridge University Press) p 57

[16] Krisch J P and Smalley L L 1988 J. Math. Phys. 291640

[17] Bayin S 1980 Phys. Rev. D 211503

[18] Cerdonio M, Prodi G A and Vitale S 1988 Gen. Rel. Grav. 2083

[19] Zurek W H 1985 Nature 317505

[20] Epstein R I, Lamb F K and Priedhorsky W C 1986 Los Alamos Science 1329 